\section{Ueber primäre Tuberculose und über die Anthrakose der Lungen und der Bronchial- drüsen.}

\section{Von Prof. Dr. Hugo Ribbert in Bonn.}

Die bei Kindern und Erwachsenen außerordentlich zahlreichen Fälle, in denen sich eine durch Verkäsung oder Verkalkung gekennzeichnete isolierte Tuberculose der bronchialen Drüsen findet, sind für die Frage nach der Eintrittspforte der Bacillen von größter Bedeutung. Sie führen ohne weiteres zu der Auffassung, daß es sich um ein Eindringen von den Respirationswegen und den Lungen aus handelt. In diesem Sinne habe ich mich mehrfach, zuletzt in dieser Wochenschrift 1904, No. 28 ausgesprochen. Nun hat man demgegenüber geltend gemacht, daß auch in diesen Fällen die Bacillen durcb den Verdauungstractus, und zwar entweder im Rachen oder im Darm aufgenommen sein könnten. Aber es ist ausgeschlossen, daß bei isolierter Bronchialdrüsentuberculose die Infektion die cervicalen und mesenterialen Drüsen übersprungen haben sollte. Sind aber auch sie tuberculös, so ist darin noch durchaus kein Beweis gegeben, daß ihre Erkrankung primär und die der bronchialen sekundär ist. Im Gegenteil, es geht weder, wie Beitzke ${ }^{1}$ ) nachgewiesen hat, ein Weg von den cervicalen Drüsen zu denen des Lungenhilus und der Lunge selbst, noch kann die Infektion von der mesenterialen ohne weiteres auf die bronchialen übergehen. Denn an diesen führt der Ductus thoracicus vorüber und es ist willkürlich, anzunehmen, daß Bacillen aus ihm seitlich gegen den Lymphstrom in die Bronchialdrüsen gelangen sollten. Bei sehr schwerer Tuberculose am Halse und im Mesenterium kann die Erkrankung allerdings Schritt für Schritt auch auf benachbarte Drïsen und so schlieblich auf den Lungenhilus sich ausbreiten. Aber das ist zweifellos selten. Meist kann man leicht nachweisen, daß die Bronchialdrüsentuberculose der Mittelpunkt ist, von dem aus nach oben und unten hin die Veränderungen der Drüsen an Intensität abnehmen. Eine gleichzeitig vorhandene Tuberculose am Hals und im Mesenterium ist dann als die Folge einer doppelten, vom Verdauungs- und vom Respirationstractus aus erfolgten Infektion anzusehen. Für den Darm habe ich das an genannter Stelle hervorgehoben. Es ist ja nicht überraschend, vielmehr selbstverständlich, daß die in der Luft suspendierten Bacillen zum Teil auf die Schleimhäute des Rachens und des Darmes gelangen und von hier aus aufgenommen werden können. Freilich werden sie in die Darmwand ungleich seltener eindringen, als in die Wandungen der Atemwege, denn sie werden sicherlich zum größten Teil mit dem Kote wieder entleert. Aber bei genügender Menge machen sie die primäre Darm- und Mesenterialdrüsentuberculose, die nach den neueren Untersuchungen ${ }^{2}$ ) immerhin häufiger ist, als man lange Zeit angenommen hat. Sie tritt aber prozentual durchaus in den Hintergrund gegenüber der Infektion durch die Respirationswege, die ja nicht nur bei isolierter Bronchialdrüsentuberculose, sondern auch bei gleichzeitiger, auf anderm Wege nicht $\mathrm{zu}$ erklärender Lungenerkrankung angenommen werden muß. Daran werden wohl auch die Mitteilungen Schlossmanns ${ }^{3}$ ) nichts ändern, der in dem Magen wenige Tage alter Tiere eingebrachte Bacillen schon nach einigen Stunden in den Lungen wiederfand und annahm, daß die Bacillen die Mesenterialdrüsen passierten, durch den Ductus thoracicus in das Blut und so in die Lungen gelangten. Von allem anderen abgesehen, ließe sich ja die menschliche isolierte Bronchialdrüsentuberculose auf diese Weise nicht verstehen.

Beim Tier mag der Darm häufiger als beim Menschen in Betracht kommen. Darauf deuten die Versuche von Barte ${ }^{4}$ ) hin, der Meerschweinchen dadurch infizierte, daß er sie in den Wohnungen von Phthisikern unterbrachte und sah, dab sie hauptsächlich vom Darm aus erkrankten. Aber das beweist

1) Virchows Archiv Bd. 184. - 2) Slehe die jüngste Arbeit von I psen, Berliner klinische Wochenschrift 1906, Bd. 24. - 3) Detutsche medizinische Wochenschrift 1906, No. 27. - 4) Wiener klinische Wochenschrift 1906, No. 7 th. 8 nichts für den Menschen. Denn die Tiere kommen in ganz andere Berührung mit dem Schmutze des Erdbodens, sie werden viel mehr Bacillen in die Mundhöhle bringen als der Mensch, und der in der Luft suspendierte Staub wird viel reichlicher in den engen ersten Wegen der Nase, des Mundes und des Rachens niedergeschlagen und demgemäB vom Verdauungstractus aus wirksam werden. Man sollte überhaupt die Tierversuche nicht $\mathrm{zu}$ sehr den Infektionsbedingungen des Menschen an die Seite stellen. Nur er, in erster Linie der bei ihm erhobene pathologisch-anatomische Befund kann uns Aufschlub über die für ihn in Betracht kommenden Eingangspforten geben. Unter ihnen nehmen aber die Atmungsorgane weitaus die erste Stelle ein. Die folgenden Auseinandersetzungen sollen dafür weitere Belege bringen.

In den Fällen von isolierter Bronchialdrüsentuberculose sind die übrigen Organe, insbesondere die Schnittflächen der Lungen, frei von tuberculösen Veränderungen. Ich nahm früher an, daß die Lungen dann überhaupt intakt seien, habe mich aber überzeugt, dal es sehr oft nicht der Fall ist. Wenn man nämlich die Pleuraflächen mit den Augen absucht oder mit den Fingern abtastet, stößt man hier oder dort, in diesem oder jenem Lappen in der Lunge, zu der die veränderte Bronchialdrüse gehört, oder in der anderen, auf derbe subpleurale Knötchen, auf eins, zwei oder selten auch mehrere. Sie sind stecknadelkopf- bis erbsengrob, bei Kindern verkäst oder verkalkt, bei Erwachsenen meist mehr oder weniger schwarz gefärbt, anthrakotisch.

Diese Knötchen entsprechen den subpleuralen Lymphdrüsen, die am genauesten von Heller ${ }^{1}$ ) beschrieben wurden. Das geht aus ihrer Lagerung hervor, läft sich aber manchmal mikroskopisch noch dadurch nachweisen, daß die Lymphdrüsenstruktur teilweise erhalten ist. Neben den einzelnen pathologisch veränderten Drüsen findet man auf der Lungenoberfläche zerstreut andere, die außer Kohleeinlagerung keine Abnormitäten aufweisen.

Daß die Erkrankung dieser Gebilde tuberculöser Natur ist, kann dann nicht zweifelhaft sein, wenn sie verkäst oder wie die Bronchialdrüsen in typischer Weise verkalkt sind.

Histologisch freilich kann man den tuberculösen Charakter meist insofern nicht mehr nachweisen, als die typischen geweblichen Produkte, die Tuberkel mit ihren Riesenzellen fehlen und auch Tuberkelbacillen nicht mehr nachweisbar sind. Das ist aber nicht auffallend, denn die tuberculösen Neubildungsprozesse sind abgelaufen und in Narbengewebe übergegangen. Das gilt auch für die meisten fest verkreideten Lymphdrüsen. Wollte man ihren tuberculösen Charakter aus der histologischen Struktur entnehmen, so käme man nicht weit. Trotzdem zweifelt, wie u. a. Hanau und Schlenker ${ }^{2}$ und besonders 0 . Naegeli ${ }^{3}$ ) hervorgehoben haben, kaum jemand daran, daß hier „geheilte" Tuberculose vorliegt. Und das ist um so sicherer, als sich gewöhnlich ein ausreichendes Kriterium findet, nämlich die Nekrose, der Käse, an den der Kalk gebunden ist. Wenn man diesen auflöst, so sieht man kleinere und größere, einzelne oder mehrere nekrotische Felder, die in derbes narbiges Bindegewebe eingeschlossen sind. Der Käse ist häufig durch nädel- und spießförmige Lücken ausgezeichnet, die früher vorhandenen Fettsäurenadeln entsprechen. Alles das findet man außer in den Lymphdrüsen und den subpleuralen Knötchen auch in den in derbes Narbengewebe eingeschlossenen, verkreideten "geheilten" Herdchen der Lungenspitzen.

Manchmal kommt zu diesen histologischen Bildern noch etwas hinzu, was wir auch sonst bei Verkalkung, aber vor allem bei der des Käses sehen, die Knochenbildung in dem Rande. Aber sie hat für uns hier kein weiteres Interesse.

Die Bedeutung der subpleuralen Kalkknötchen ist also völlig klar. Es handelt sich um abgelaufene Tuberculose. Es gibt aber andere Herdchen, die ihre Aetiologie nicht so ohne weiteres verraten.

Bei Erwachsenen nämlich, umso ausgesprochener, je älter sie sind, finden sich an den gleichen Stellen Knötchen, die man auf den ersten Blick als anthrakotische bezeichnen wird, die sich derb anfühlen und manchmal auch verkalkt sind. Sie

1) Deutsches Archiv fitr klinische Medizin No. 55. - 2) Virchows Archiv Bd. 134 $-3)$ ibid. Bd. 100 
finden sich ebenfalls einzeln oder zu mehreren, hier oder dort, ohne dab in den Lungen Tuberculose oder schwerere anthrakotische Veränderungen nachweisbar wären, nicht selten aber gleichzeitig neben solchen Prozessen des Lungengewebes. Im ersteren Falle sind sie aber stets vergesellschaftet mit verkalkten oder mit hochgradig anthrakotisch indurierten oder auch trocken nekrotisch erweichten Lymphdrïsen.

Soweit diese anthrakotischen Knötchen verkalkt sind, wird wohl allgemein ihre tuberculöse Natur zugegeben werden, auch wenn histologisch nicht die gleichen Befunde wie dort vorhanden sind. Denn nicht immer trifft man Käse als Träger der Verkalkung an. Nicht selten liegt der Kalk in dem sklerotischen, dichten, kernlosen, oft mit Fettsäurenadeln oder deren Resten durchsetzten Narbengewebe der mittleren Abschnitte der Knötchen, ohne daß hier eine typische Nekrose mit molekularem Zerfall nachweisbar wäre. Die verkalkten Abschnitte sind dabei wesentlich ärmer an Kohle, als die peripheren Knötchenteile, die allein dem Begriffe der anthrakotischen Induration entsprechen. In solchen Herdchen ist also ursprünglich die Tuberculose weniger intensiv gewesen, als in den zuerst beschriebenen, es kam nicht zur Entwicklung größerer Käsemengen, dagegen zur bald einsetzenden Vernarbung und damit einhergehenden, zunehmenden Einlagerung von Kohle.

Weniger leicht $\mathrm{zu}$ beurteilen sind die Knötchen ohne Verkalkung. Um sie zu verstehen, müssen wir zunächst im allgemeinen auf die Beziehungen zwischen Tuberculose und Anthrakose eingehen.

Man ist meist der Meinung, daß die Kohleansammlung allein hochgradige Veränderungen der Lungen bewirken könne. Erweichung bis zur Cavernenbildung und weitgehende anthrakotische Verdichtung werden aus ihr abgeleitet, ohne daf Tuberculose im Spiele sein soll.

Das halte ich nicht für richtig. Wenn wir uns fragen, in welchem Umfange die Kohlenstaubeinatmung die Lungen beteiligen muß, so kann die Antwort nur so lauten, daß das Organ, da der Staub ja überall hinkommt, im großen und ganzen gleichmäßig erkranken muß, allerdings nicht in dem Sinne, daß das Gewebe sofort diffus grau und schwarz würde, sondern so, daß die Kohle sich in alle die zahllosen, kleinen, lymphatischen Herdchen, bzw. in deren nächste Umgebung und weiterhin in Verfolgung der Lymphbahnen ablagert, sodaß die schwarzen, zackigen Flecke der Schnittfläche und die schwarzen Netze der Pleura entstehen. Erst mit fortschreitender Einatmung werden auch die zwischen den Herden gelegenen Teile pigmentiert und schlieflich ist die Lunge überall so gefärbt. Solche Fälle sind aber weniger häufig als die anderen.

Weitaus überwiegend tritt die Anthrakose in Form der schiefrigen Induration herdförmig auf in kleineren und größeren, strahligen, derben, hier oder dort, besonders im Oberlappen und hier wieder in der. Nähe der Spitze sitzenden Bezirken. Dann kann es sich nicht um reine Anthrakose handeln, mag nun das Gewebe außerhalb der Herde diffus schwarz gefärbt, aber noch lufthaltig, oder mag es nur schwarz gefleckt oder, was nicht ganz selten ist, fas t $\mathrm{k}$ ohlefrei sein.

Denn wie sollte es möglich sein, daß die eingeatmete Kohle in die jetzt verdichteten Partien in besonders großer Menge gelangte, während sie doch in alle anderen Abschnitte ebenso eindringen konnte? Das mulf seinen besonderen Grund haben, und der ist darin zu suchen, daß dem anthrakotischen Herde entsprechend schon vor der Kohleeinlagerung ein pathologischer Zustand vorhanden war, der dann, weil der eingeatmete Staub nicht mehr, wie aus normalen Lungenabschnitten größtenteils wieder entfernt werden kann, zu seiner immer dichteren Ablagerung führt. Es ist also nicht so, dab die Kohle die Verdichtung erst bewirkt, sondern so, daß sie die entzündliche Bindegewebezunahme bereits vorfindet und in ihr liegen bleibt, wobei sie dann vielleicht ihrerseits zur Induration beiträgt. Nur so läbt sich das herdförmige Auftreten der schiefrigen Anthrakosis verstehen.

Nun erhebt sich die Frage nach der Aetiologie der Entzündungsherde. Es kann Verschiedenes in Betracht kommen, so Syphilis, unvollkommen resorbierte Bronchopneumonien, Abscesse oder Infarkte, aber es ist ohne weiteres klar, daß diese Erkrankungen und andere, die noch seltener sind, quanti- tativ keine große Rolle spielen. Nur die Tuberculose kann die wichtigste Grundlage abgeben. Schon diese Ueberlegungen führen uns also dahin, in den meisten anthrakotischen, herdförmigen Indurationen der Lunge ausgeheilte tuberculöse Erkrankungen zu sehen.

Für diese Auffassung lassen sich ferner folgende Erfahrungen verwerten. Manchmal sind wir imstande, in den makroskopisch scheinbar rein anthrakotischen Herden histologische Kriterien von Tuberculose-Granulationsgewebe mit Riesenzellen aufzufinden, oder es sind in der indurierten Substanz noch wechselnde Mengen von Alveolen erkennbar, aber ausgefüllt mit einem kernlosen nekrotischen Exsudat, dem Rest einer früheren käsigen Pneumonie, die in dem indurierten Gewebe fest eingeschlossen liegen blieb. Das gilt insbesondere auch für die manchmal umfangreichen anthrakotischen Herde, in denen sich mehr oder weniger große, einzelne oder mehrere, fetzige oder auch glattwandige, bronchiektatische Cavernen finden. Es ist zweifellos ein Irrtum, wenn man diese Höhlenbildungen von der Kohle allein abhängig gemacht hat. Wie sollte es auch möglich sein, daf sie zum Zerfall führte! Es handelt sich immer um eine früher einmal deutlich erkennbare, dann allmählich in schiefrige Induration übergegangene Tuberculose.

In anderen Fällen ist die Tuberculose neben den Herden schon mit bloßem Auge nachweisbar und dann nach abwärts in den Lungen meist noch mehr ausgesprochen. Dann kann an der Bedeutung der anthrakotischen Knoten als ausgeheilter tuberculöser Prozesse kein Zweifel sein. Oder man bemerkt auf der Schnittfläche der Herde und der übrigen Lunge nichts von Tuberculose, aber auf weiteren Durchschnitten stöbt man in ihnen schließlich auf kleinere oder größere käsige Einschlüsse.

Wieder andere Bezirke lassen weder für das bloße Auge noch für das Mikroskop für Tuberculose sprechende Befunde hervortreten. Sie sind aber im übrigen genau so gebaut wie jene Herde von unzweifelhaft tuberculöser Genese, und nichts spricht dagegen, daß auch sie durch Vernarbung früherer tuberculöser Prozesse entstanden. Mit der Vorstellung aber, dab sie allein der Kohle ihr Zustandekommen verdanken, steht nicht selten der Umstand in Widerspruch, daß die Kohle gerade dort, wo die Induration am festesten ist, in wesentlich geringerer Menge liegt, als in den peripheren, schwächer oder kaum verdichteten Abschnitten. Wenn trotzdem die kohleärmeren Partien makroskopisch auch intensiv schwach aussehen, so liegt das daran, dal man in das sklerotische homogene Gewebe hineinsieht und deshalb nicht nur die an der Schnittfläche befindliche Kohle wahrnimmt, sondern auch die der tieferen Schichten.

Alle diese Tatsachen, daß also die Anthrakose in Herden auftritt, während man diffuse Veränderungen erwarten müßte, daß sie sich sehr oft ohne weiteres als abgelaufene Tuberculose zu erkennen gibt, daß sie sich histologisch in keinem Punkte von unzweifelhaften Vernarbungen früherer Tuberculosen unterscheiden, lassen den Schluf gerechtfertigt erscheinen, daß die meisten anthrakotischen Herde die Endstadien ausgeheilter tuberculöser Erkrankungen darstellen.

Diese Ueberlegungen und Erfahrungen gelten aber auch für die Bronchialdrüsen. Soweit sie, was außerordentlich oft und viel häufiger, als in den Lungen der Fall ist, anthrakotisch und zugleich verkäst oder besonders verkalkt sind, wird man sie ohne weiteres für tuberculös halten. Bei den anderen dagegen, die nicht verkreidet, sondern nur anthrakotisch induriert sind, aber sehr gewöhnlich auf der Schnittfläche entweder schwarzgraue, trockene, verwaschene Nekrose oder auch Erweichungen zeigen, müssen Schlubfolgerungen Platz greifen. Zunächst einmal sind sehr oft nicht alle Drüsen induriert, manchmal nur eine, während die anderen, ebenso viel Kohle enthaltenden weich und zellreich blieben. Das zeigt allein schon, daß die Kohle für sich die Induration nicht macht, daß vielmehr besondere Umstände hinzukommen oder vielmehr voraufgehen müssen. In demselben Sinne ist es $\mathrm{zu}$ verwerten, wenn eine Drüse nur zum Teil anthrakotisch, zum andern weich geblieben ist. Man findet ferner nicht selten Anthrakose einer Drüse und in ihr zugleich einen Kalkknoten oder 
es sind mehrere anthrakotisch, andere in wechselndem Umfange fest verkalkt, oder es besteht wohl auch neben der Anthrakose eine frischere, allmählich in den indurierten, vernarbten Zustand übergehende tuberculöse Struktur. Nehmen wir ferner hinzu, dab die Anthrakose, die man auf die Wirkung der Kohle allein beziehen möchte, sich in nichts histologisch von der aus Tuberculose hervorgegangenen, also z. B. neben Verkalkung vorhandenen unterscheidet, und endlich, daß auch in den Drüsen das eben für die Lunge hervorgehobene Mißverhältnis zwischen Induration und Menge der Kohle besteht, dab also letztere oft spärlicher ist, als in nicht anthrakotischen Drüsen, und zwar offenbar deshalb, weil sie in das narbig verdichtete Gewebe nicht mehr hineingelangte, so muß man auch hier zu dem Schlub kommen, daß die anthrakotischen Indurationen der Bronchialdrüsen der Mehrzahl nach - soweit nicht andere, aber in den Drüsen jedenfalls seltene Entzündungsprozesse in Betracht kommen - auf Tuberculose beruhen.

$\mathrm{Zu}$ denselben Schlußfolgerungen führt die Untersuchung der subpleuralen anthrakotischen Knötchen. Soweit sie verkalkt sind, wurden sie schon besprochen. Bei den nicht verkalkten muß Tuberculose vorausgesetzt werden, weil gewöhnlich die Induration nur einzelne getroffen hat, während andere, ebenso stark kohlehaltige weich und zellig bleiben, weil neben ihnen andere Knötchen Kalkablagerungen zeigen, weil ihr Kohlegehalt oft so gering ist, daß auf ihn die Verdichtung nicht bezogen werden kann, weil sie ferner meist mit verkästen und verkalkten Bronchialdrüsen (oder mit ausgeheilten tuberculösen Lungenerkrankungen) vergesellschaftet sind, weil endlich die indurierten Gebilde in allen Fällen völlig gleich gebaut sind.

Damit kommen wir auf unsere Ausgangsbetrachtungen zurück. Die neben tuberculösen Bronchialdrüsen in sonst gesunden Lungen häufig vorkommenden subpleuralen, aus den kleinen Lymphdrüschen hervorgegangenen Knötchen sind überwiegend tuberculöser Natur. Bei den verkästen und verkalkten ist das ohne weiteres klar, bei den anderen aus Schluffolgerungen zu entnehmen. In ihrem Vorhandensein liegt nun aber ein wichtiges Kriterium für die Eingangspforte der Tuberculose. Es wird kaum jemand die subpleuralen Knötchen von Bacillen ableiten wollen, die vom Verdauungskanal eindrangen, sie sind sicherlich von inhalierten Bacillen abhängig, die gerade wie die Kohle zum kleineren Teil auf dem Wege der Lymphbahnen in die subpleuralen Lymphdrüsen, zum anderen Teil in die Bronchialdrüsen gelangten.

Die subpleuralen Knötchen liefern also den Beweis für eine a ërogene Infektion der Lungen, damit aber zugleich auch eine Grundlage für die Entstehung der Bronchialdrüsentuberculose anf aërogenem Wege. Das gilt übrigens auch für kleine Knötchen, die sich bei scheinbarer isolierter Hilustuberculose hier und da auch in der Lunge neben den Bronchien finden, denselben Bau haben wie die subpleuralen und auch wie sie aus kleinsten Lymphdrüschen hervorgehen.

Aber die Bronchialdrüsenerkrankung ist selbstverständlich nicht nur abhängig von der Entstehung der Herdchen unter der Pleura oder der intrapulmonal neben den Bronchien gelegenen, sie kann vielmehr auch für sich allein zustande kommen. Sie wird ja häufiger isoliert angetroffen, als in Verbindung mit jenen Knötchen. Die Bacillen können also durch die Respirationswege aufgenommen werden, ohne in ihnen Spuren $\mathrm{zu}$ hinterlassen, genau so, wie sie dırch den Darm, ohne ihn zu verändern, hindurchtreten können.

Die subpleuralen Knötchen haben aber noch ein weitergehendes Interesse. Sie zeigen, daß aspirierte Bacillen durchaus nicht notwendig in den Spitzenabschnitten der Lungen zur Ansiedelung gelangen müssen. Aber noch mehr, sie lehren auch mit Bestimmtheit, daß Bacillen, die unzweifelhaft in das Lungengewebe gelangten, trotzdem in ihm wirkungslos bleiben und erst nach Ueberführung in die subpleuralen Herdchen Veränderungen hervorrufen können. Die Bronchialdrüsentuberculose ist in diesem Sinne nicht ebenso sicher beweisend, weil sie ja auch durch Bacillen bedingt sein könnte, die von der Schleimhaut der Bronchien aufgenommen wurden, ohne in das eigentliche Gewebe der Lungen zu gelangen.

Die hier mitgeteilten Untersuchungen sind aber endlich von Bedeutung für die Häufigkeit der latenten Tuberculosedes Menschen. Wenn zu den als solchen sicher nachweisbaren Tuberculosen, besonders der Bronchialdrüsen, nun auch noch solche Fälle von anthrakotischen Indurationen kommen, die nicht durch gleichzeitige, manifeste Tuberculose ohne weiteres gekennzeichnet sind, so steigt damit der Prozentsatz derjenigen Individuen, die als sicher tuberculös infiziert anzusehen sind. Die hohen Zahlen von Naegeli und anderen erhalten dadurch eine größere Sicherheit.: Denn man hat ja gerade betont, daß anthrakotische Narben als nicht genügend gesichert aufer Betracht gelassen werden müßten, und hat so den Prozentsatz von 95 auf 70 und 60 herabsetzen zu sollen geglaubt. Ich halte das für durchaus unberechtigt, aber ich möchte hervorheben, daß in einer bestimmten, möglichst beträchtlichen Höhe des Prozentsatzes, den ich auch jetzt noch auf mindestens 90 veranschlage, garnicht, oder doch nicht allein der große Wert jener Untersuchungen liegt. Er ist vielmehr in gleich zu erwähnendem Sinne auch dann schon vorhanden, wenn erheblich mehr als die Hälfte aller Menschen eine tuberculöse Infektion erfahren hat. Das ist aber nach den Obduktionsergebnissen absolut sicher. Freilich hat man gegen die anatomischen Befunde eingewandt, daß die am Leichenmaterial der Krankenhäuser gewonnenen Resultate für die Bevölkerung im ganzen nicht maßgebend seien. Das mag richtig sein, setzt aber die Bedeutung jener Zahlen nicht wesentlich herab. Denn für die Volkskreise, aus denen sich die Patienten der Krankenhäuser rekrutieren - und das ist zweifellos die weitaus überwiegende Mehrzahl, mindestens $90 \%$ der Menschen - treffen sie zu. Ob sie indessen für die besser situierten Kreise namhaft geringer sind, müßte erst noch festgestellt werden. Denn die Sterblichkeitsziffern, die bei besserer $\mathrm{Hy}$ giene naturgemäß tiefer sind, lassen einen Schlub auf die latente Tuberculose nicht zu. Aber es mag ja, da bei geringerer Infektionsgelegenheit mehr Menschen frei von Bacillen bleiben werden, wahrscheinlich sein, daß jene Zahlen für die wohlhabenden Bevölkerungsklassen wirklich zu hoch sind. Dann beeinträchtigt das doeh nicht den Wert jener Resultate, der darin besteht, daß weitaus die meisten Menschen einmal mit Tuberculose infiziert wurden, dab sie aber nur zum kleineren Teil wirklich erkrankten, zum größeren relativ immun waren. Nur disponierten Menschen können die Tuberkelbacillen ernstlich gefährlich werden. Und dagegen kann man nicht etwa, wie es geschehen ist, einwenden, daß die nur latent erkrankten Menschen nur avirulente Bacillen eingeatmet hätten. Denn es ist undenkbar, daß mehr als die Hälfte der Menschen ausschließlich nur Gelegenheit gehabt haben sollte, abgeschwächte Bacillen in sich aufzunehmen. 